\title{
A GIS-based assessment of Tibet's potential for pumped hydropower energy storage
}

\author{
$\mathrm{Xu} \mathrm{Lu}{ }^{\mathrm{a}, 1, *}$, Siheng Wang ${ }^{1, \mathrm{c}, 1}$ \\ ${ }^{a}$ Clark University, 950 Main Street, Worcester, Massachusetts, United States, 01610 \\ ${ }^{b}$ State Key Laboratory of Remote Sensing Science, Institute of Remote Sensing and Digital \\ Earth, Chinese Academy of Science, Beijing, China, 100101 \\ ${ }^{c}$ University of Chinese Academy of Sciences, Beijing, China, 100049
}

\begin{abstract}
The cost reduction of photovoltaic (PV) module makes solar energy a promising renewable energy for large-scale electricity generation, further controlling the green house gas emissions. A primary obstacle for the connection of PV generation into electric grid is its poor stability, due to the variation of solar radiation, which determines the PV output. Pumped hydroelectric storage (PHS) is an efficient energy storage method to stabilize the intermittent PV output. Tibet, where solar radiation is in abundance, presents an opportunity to install PV stations across China, and unified construction of PHS is necessary for gridconnected utilization of the solar energy there. The objectives of this study are to evaluate the PHS potential in Tibet and to provide promising locations of the PHS stations, through Geographic Information Science (GIS) analyses. A review of the existing GIS methods (T1 - T7) for PHS site selection was firstly given. Two new GIS models (S1 and S2) appropriate for Tibetan area were proposed then, and the T1, S1 and S2 were considered for this assessment. Results showed that the total PHS potential in Tibet was about 997.2 GWh, 946.2 GWh and 2552.0 GWh under T1, S1 and S2, respectively. All the promising sites were mapped, and an assessment of these sites were made according to their distances to grid connections. The results were supposed to benefit the planning of the PHS facilities in Tibet.
\end{abstract}

Keywords: pumped hydropower storage, Tibet, GIS, potential assessment, spatial analysis

\footnotetext{
This document is a collaborative effort.

${ }^{*}$ Corresponding author

Email address: luxurdfz@sina.com (Xu Lu)

${ }^{1}$ These two authors are equally contributed to the work.
} 


\section{Introduction}

Tibet's annual average solar radiation reaches $6000-8000 \mathrm{MJ} \cdot \mathrm{m}^{-2} \cdot$ year $^{-1}$ , ranking the first in China and the second worldwide after the Sahara desert [1]. Arid deserts account for about 18\% of Tibet's land area in 2008 [2]. Thus, Tibet is supposed to be one of the most suitable areas to exploit solar energy in China because of the abundance of solar radiation and ample space for construction of photovoltaic (PV) generation stations. Meanwhile, the state's government has launched a series of projects concerning domestic PV industry. In June 2014, the General office of the state council of the people's republic of China [3] issued No.31 document ([2014]31), which set a aim of $100 \mathrm{GW}$ of PV power installed in 2020 and emphasized on accelerating the development of solar power. By the end of June 2015, the total installed PV capacity of China was 35.78 GW. However, the total installed PV capacity of Tibet was only $150 \mathrm{MW}$, far behind the other remote provinces or autonomous regions in China, like Qinghai (4.70 GW), Ningxia (2.39 GW), Inner Mongolia (4.03GW), Xinjiang (5.70 GW), and Gansu (5.78 GW) [4]. Apart from the harsh natural environment, another main obstacle for the utilization of Tibet's solar energy is its poor electricity infrastructure. By the end of 2014, the total installed capacity of the Tibet grid is only $1.697 \mathrm{GW}[5]$. A high penetration of renewable energy generation will have a detrimental effect on the power distribution network (e.g. widespread blackouts) because of the intermittency of the output [6]. Thus, large-scale integration of fluctuating renewable energy power into the existing Tibet grid system is not technically or economically practical for the moment. However, these problems are expected to be solved by Pumped Hydroelectric Storage (PHS) in the future.

PHS is a method of storing energy by pumping water from a lower reservoir to an upper reservoir and producing electricity by converting the water's gravitational potential energy (Figure 1). PHS accounts for more than $99 \%$ of worldwide bulk storage capacity and contributes to about $3 \%$ of global electricity generation and it is currently the only commercially-proven fuel free energy storage technology with large volume, long lifetime, relatively long discharge time and high efficiency [9]. Up to now, PHS is still one of the most costefficient options for bulk energy storage $[9,10]$. Energy storage can increase the penetration of renewable energy sources significantly by eliminating the intermittency and instability [11-14]. Coupled with renewable sources, PHS has the lowest greenhouse gas (GHG) emissions significantly less than other electrical energy storage technologies [15]. Previous studies [16-21] had exploited the feasibility of developing the hybrid system combining the photovoltaic (PV) power generation and the PHS system through mathematical model and operational principle, presenting a promising prospect.

The government of China has been consistently launching a series policies to promote the domestic development of PHS. In August 2014, the National Development and Reform Commission (NDRC) [22] issued No.1763 document ([2014]1763) and proposed a new price mechanism of PHS station. In November 2014, NDRC [23] issued No.2482 document ([2014]2482) and set a target of 100 


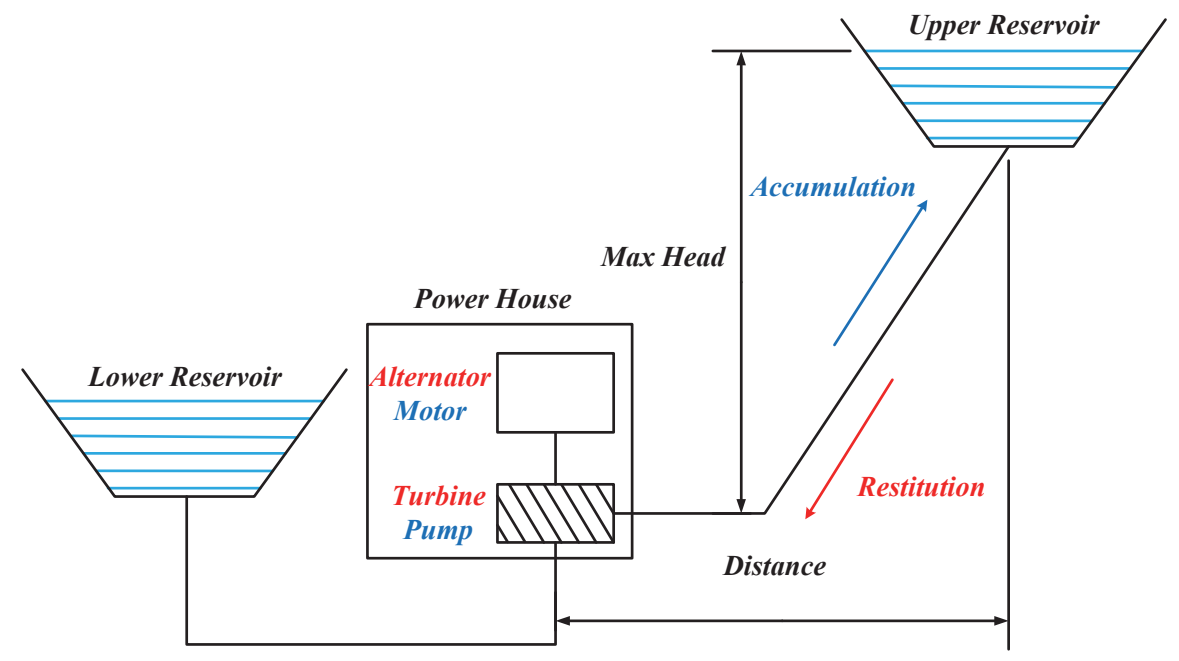

Figure 1: Schematic diagram of a pumped hydroelectric storage system $[7,8]$. The lines between reservoirs and power house are penstock.

GW of PHS by 2025, which expected to account for $4 \%$ of the total installed power capacity. In March 2015, the Central Committee of the Communist Party of China and the State Council of the People's Republic of China [24] issued No.9 ([2015]9), which aimed to establish an effective competitive market structure and market system, and to make the energy price mainly determined by the market. Once the social capital could gain profit from operating the PHS station in the market, the previous obstacles, like the management mode and price mechanism of operation and policy barriers [25], will be overcome.

Moreover, China has been continually strengthening the electricity infrastructure of Tibet's grid, which could support the development of PHS. In the past, Tibet's harsh environment and poor electricity infrastructure resulted in a few people paying attention to PHS potential in Tibet. By the end of 2013, China's total PHS capacity was 21.5 GW [23] where the capacity for Tibet was only $90 \mathrm{MW}[25,26]$ and there is no newly constructed PHS station since 2010. In the recent five years, the government built many new substations and upgraded existing substations in Tibet during the 12th Five-Year Plan to enhance the capacity of Tibet's grid. At the same time, Qinghai-Tibet grid interconnection project connected Tibet's isolated grid with northwestern grid of China in 2011 and the long-term goal of maximum power of electricity delivery is $1.2 \mathrm{GW}$ [27]. Although the project was designed to solve the electricity shortage in Tibet, it can deliver electricity to Qinghai at summer now [28]. The project shows the ability that the total installed capacity of the Tibet grid can exceed the local power consumption and export extra power to other provinces. Sichuan-Tibet 
grid interconnection project was completed in 2014 [29]. The two projects are similar and latter one can directly deliver Tibet's electricity to inland China in the future. In addition, the costs of large-scale solar power in Tibet will be close to thermal power in the near future due to the high solar radiation intensity and long sunshine duration in Tibet and a substantial drop in price of solar power equipment. Thus, capacity of Tibet's grid will expand a lot in the future and the capacity of PV generation will increase as well. The demand of using PHS to regulate PV generation would be stronger than before. If there are adequate available PHS sites in Tibet, China can exploit solar power at large scale coupled with PHS in Tibet and be less dependent on coal, reducing the GHG and pollution emissions. Thus, unified construction of PHS is necessary for grid-connected utilization of the solar energy in Tibet.

Many previous studies applied Geographic Information Science (GIS) methods to discover the latent sites for PHS, because the scarcity of available site for two large reservoirs is the most impactful constaint [30]. Connolly et al [31] developed a computer program to identify potential PHS sites based on digital terrain maps. Fitzgerald et al [32] proposed a GIS-based model to calculate theoretical potential of a large area for the development of PHS schemes from existing conventional hydropower stations and from non-hydropower reservoirs. The Joint Research Centre of European Commission [33] reported that there are seven different topological relations (Table 1) between two reservoirs and analyzed the theoretical potential sites for new PHS station in countries of the European Union under categories T1 and T2 using GIS-based model and a series of social, infrastructure and environmental constraints. Idaho National Laboratory [34] also conducted a comprehensive research assessing the theoretical potential for PHS under categories T1 and T2 in the US. Using GIS model can assess the potential for PHS more comprehensively and economically without long field trip, especially for the remote areas with harsh environment like Tibet. 
Table 1: Brief description of the different PHS topologies from the point of view of assessing PHS potential [33, 35]

\begin{tabular}{cl}
\hline Topology & Description \\
\hline T1 & Linking two existing reservoir with one or several penstock(s), \\
& and adding a powerhouse to transform them to a PHS scheme \\
T2 & Transformation of one existing lake or reservoir to PHS by de- \\
& tecting a suitable site for a second reservoir. The second reser- \\
& voir could be on a flat or non-sloping area, by digging or building \\
& shallow dams, on a depression or in a valley \\
T3 & A greenfield PHS based on a suitable topographical context: ei- \\
& ther valleys which can be closed with a dam, hill tops which could \\
& be slashed, tec. This topology is broader i.e. neither based on \\
& existing lakes or reservoirs nor assuming a flat area for building \\
& the second reservoir \\
T4 & Sea-based PHS: a greenfield PHS that uses the sea as the lower \\
& reservoir and a new nearby reservoir, or the sea as upper basin \\
& and a cavern as lower reservoir \\
T5 & Multi-reservoir systems including both PHS and conventional \\
& hydropower \\
T6 & The lower reservoir is basically a large river providing sufficient \\
& inflow into the PHS system \\
T7 & Use of an abandoned mine pit as the basis for the PHS. The \\
& methodology to be used would be similar to the T2 one \\
\hline &
\end{tabular}

The objectives of this paper are to provide a series of most promising sites for PHS stations and to assess the PHS potential in Tibet using GIS models, aiming to help overcoming the scarcity of available sites in this region. Therefore, there are two topologies considered in this paper, referred as T1 and T2 in Table 1. For T2, previous studies applying GIS models seemed to overlook some of the valuable potential sites for PHS. Flat areas for building the second reservoir was usually the situation considered, but the majority of the PHS schemes choose to construct the second reservoir through blocking a valley with a dam rather than on a flat field [33]. Moreover, for regions like Tibet with rough terrain, the main opportunity for the second reservoir lies in the second situation. Thus, a new GIS model, which is referred as S1 (S2), that is set to find the second reservoir by blocking a valley is more appropriate for Tibet to discover more potential sites.

The paper is structured as follows: Section 2 introduces the data used in this study; Section 3 provides a brief description of the methodology and tools applied; Section 4 presents the results for Tibet; Section 5 analyses the results and limitations of the study. 


\section{Data}

\subsection{Study area}

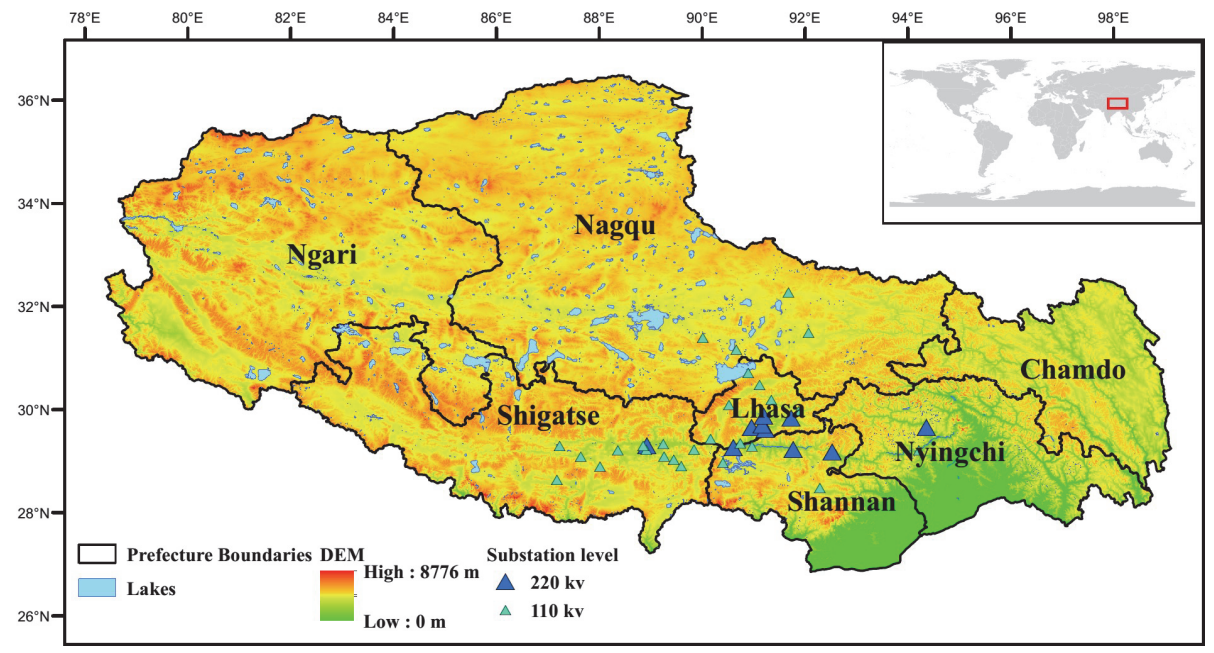

Figure 2: Study area map

The study area is the Tibet autonomous region (Tibet for short), which is a provincial region of the People's Republic of China (PRC) (Figure 2). Tibet is located in the southwest China between $78^{\circ} 25^{\prime}-99^{\circ} 06^{\prime} \mathrm{E}$ longitude and $26^{\circ} 50^{\prime}-36^{\circ} 53^{\prime} \mathrm{N}$ latitude. Its total land area is over 1.22 million square kilometers, accounting for about $12.8 \%$ of the China's total area. Tibet is the main part of the Qinghai-Tibet Plateau with an average altitudes of over $4000 \mathrm{~m}$. Tibet is known as "The Water Tower of Asia" as several major rivers of the continent have their sources there. Tibet has more than 1500 lakes and as such it has abundant water resources. Tibet has seven subareas that include Lhasa, Shigatse, Chamdo, Ngari, Nagqu, Shannan, and Nyingchi.

\subsection{DEM data}

The Advanced Spaceborne Thermal Emission and Reflection Radiometer's (ASTER) Global Digital Elevation Model (GDEM) version 2 might be the best publicly available global DEM now [36]. GDEM is better than Shuttle Radar Topography Mission's (SRTM) 3-arc-second elevation grid global DEM datasets, since it has a larger land surface coverage (between 83 degrees north latitude and 83 degrees south latitude), a finer spatial resolution (1-arc-second, about 30 $\mathrm{m}$ ), more updated data (from 2000-2010), and higher accuracy [37, 38]. Thus, the topology derived from the GDEM would be accurate enough for the sites selection in this study. 


\subsection{Hydrography data}

The SRTM Water Body Data files are a by-product of the data editing performed by the National Geospatial-Intelligence Agency (NGA) to produce the finished SRTM Digital Terrain Elevation Data Level 2 product and the spatial resolution of the data is 1-arc-second (approx. 30m) [39]. Since there is no publicly available hydrography data, SRTM Water Body Data was used to depict all water bodies including the man-made reservoirs and natural lakes, in Tibet.

\subsection{Grid infrastructure data}

The distance from theoretical potential sites to different level grid connections could show the feasibility to build a new PHS station since the construction of a new transmission line is costly [8], especially in Tibet due to high altitude. Thus, the closer the PHS station is located to the existing substations (grid connections), the lower the costs of integration to the grid. In this study, all substation sites within Tibet are extracted from a blue map of State Grid in 2011 and the shapefile is manually created by geo-referencing these sites in Google Earth (see Figure 2).

\section{Methodology}

The site selection strategy should be technically and economically feasible based on Tibet's terrains. Considering Tibet's complex terrains, comprising a lot of mountains, valleys, and lakes, two topologies were considered in this study:

1. Finding potential PHS sites based on linking two existing lakes (T1).

2. Taking an existing lake and exploring the surrounding valley for a new reservoir (T2) to discover a potential site to build PHS.

The platform used in this study is exclusively ArcGIS 10.2. All the algorithms applied can be found in Arctoolboxes and the model builder module of the software was used to process batch data. The entire processing time took several hours using a single PC, based on the parameters of this study. This is a very convenient and time-saving methodology, which can be applied to other case studies.

\subsection{Data preprocessing}

The main objectives of the data preprocessing stage were to extract the elevation of all water bodies and to calculate the surface slope within the study area. The "zonal statistics as table" tool was used to obtain the elevation of each lake, which was calculated as the average elevation of each pixel. The elevation of each lake was regarded as the average elevation within each lake's extent. The slope of Tibet was calculated using the "slope" tool. 


\subsection{Category T1 — linking two existing water bodies}

Linking two existing water bodies was considered under category $\mathrm{T} 1$ for this study. Figure 3 shows the flowchart of site selection under T1 and all constraints in this model are listed in Table 2. The processing includes three steps:

\subsubsection{Find pairs of lakes}

The search radius was set at $10 \mathrm{~km}$ to generate a table recording the adjacent water bodies around the specified water body using "Generate Near Table" tool. The distance threshold $(10 \mathrm{~km})$ was set as an estimated value, which is close to the horizontal distance $(8.6 \mathrm{~km})$ between two natural reservoirs of the Yangzhuoyong Lake Pumped-storage Power Station, which is the only PHS station in Tibet. This step would identify pairs of water bodies within a distance below the threshold. A maximum limit could be set for the closest pairs of water bodies to reduce the number of pairs identified and computation time.

\subsubsection{Apply restrictions}

Unsuitable pairs of water bodies according to constraints under T1 (Table 2) were eliminated and the remaining lake pairs were theoretical potential sites. There is a minimum max head threshold of $500 \mathrm{~m}$ that is recommended by the government of China [23]. A minimum water body area of $60000 \mathrm{~m}^{2}$ was used, which is the rounded value of the smallest area within the water body data set. The ratio of max head to distance (Figure 1) in each pair of water bodies was calculated and it is a subjective threshold to reduce the number of potential sites.

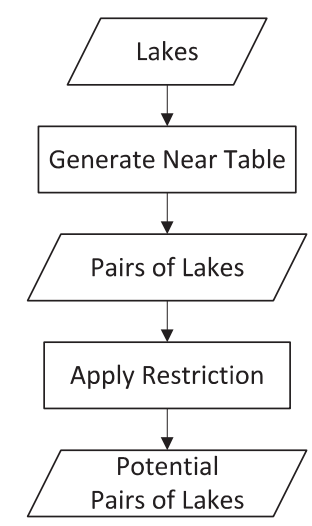

Figure 3: Methodological flowchart under category T1 
Table 2: Constraints under category T1

\begin{tabular}{ll}
\hline Description & Value \\
\hline Search radius & $10 \mathrm{~km}$ \\
Maximum number of closest catches & 10 \\
Minimum head & $500 \mathrm{~m}$ \\
Minimum water body area & $60000 \mathrm{~m}^{2}$ \\
Minimum ratio of head to distance & 0.1 \\
\hline
\end{tabular}

\subsection{Category T2 - linking an existing water body and a newly built reservoir}

In Tibet, many water bodies are surrounded by valleys, which can be converted to the second reservoir. In this study, only linking an existing lake and a newly built reservoir, which is built by closing a valley with a dam, was considered under category T2. Table 3 details the constraints of the topology model.

\begin{tabular}{ll}
\multicolumn{2}{c}{ Table 3: Constraints under category T2 } \\
\hline Description & Value \\
\hline Search radius & $5 \mathrm{~km}$ \\
Buffer interval & $500 \mathrm{~m}$ \\
Minimum head & $500 \mathrm{~m}$ \\
Minimum lakes area & $60000 \mathrm{~m}^{2}$ \\
Minimum slope & 10 degrees \\
Maximum difference of elevation & $-70 \mathrm{~m}$ \\
Minimum flow accumulation & 100 pixels \\
\hline
\end{tabular}

\subsubsection{Extract drainage lines}

The hydrology toolset in ArcGIS was applied to simulate the drainage lines [40]. Since valley lines have drainage patterns [41], drainage lines could be viewed as valley lines for the following procedure.

To extract drainage lines as thoroughly as possible, especially in the places near mountaintops, a low threshold should be specified on the raster derived from the Flow Accumulation tool. In this study, the threshold was set as 100 pixels based on experiments' results. This threshold means at least 100 pixels should contribute to the flows of each pixel on the drainage lines.

\subsubsection{Generate topologically potential site points}

Figure 4 illustrates the design to create potential topological site points. The topological restrictions for the potential points are in a valley and within $5 \mathrm{~km}$ of the existing lakes. The search radius is based on previous studies [33, 34], because setting a distance that is too large is not practical for construction. To estimate the number of potential site points within $5 \mathrm{~km}$ of the existing lakes, the "Multiple Ring Buffer" tool was used to generate eleven buffers from $500 \mathrm{~m}$ to $5000 \mathrm{~m}$ evenly. The buffer interval is a subjective variable, because $500 \mathrm{~m}$ is a compromise between complete potential site points and computing time in this 
study. The intersections of buffers and drainage lines were then identified. Since the drainage lines were assumed as valley lines, the intersections were supposed to be potential site points. Each point was determined to be either an upper or a lower reservoir. Finally, the distance (from each point to corresponding lake), the slope and the head of each point were calculated.

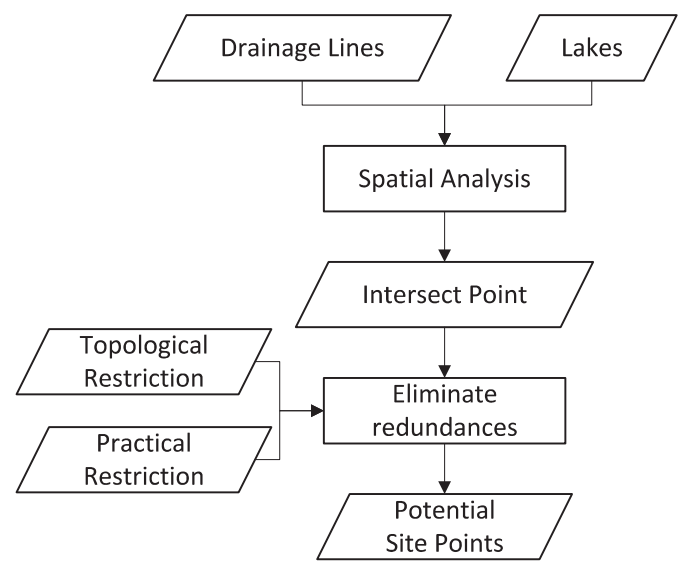

Figure 4: Flowchart to generate the potential site points

\subsubsection{Eliminate redundant points}

The low threshold value (100 pixels) that was set in 3.3 .1 would create too many redundant drainage lines, which are not located in actual valleys (Figure $5(\mathrm{a}))$. The unnecessary intersections on redundant drainage lines should be eliminated. These valleys are usually deep and wide, therefore, the distance between two nearby ridge lines is generally greater than $1.5 \mathrm{~km}$ (approx. 50 pixels) and the depth of valleys is usually greater than $70 \mathrm{~m}$. Using these parameters, the "Focal Statistics" tool was applied to smooth the DEM image. The neighborhood was defined as a circle and the radius (half the distance between two nearby ridges) was assigned a value of 25 pixels. The result of the mean filter (smoothing) were subtracted from original DEM. The pixels with a value lower than $-70 \mathrm{~m}$ in the output image was assumed to be a valley (Figure $5(\mathrm{~b})$ ). Finally, selecting these intersections located in valley areas, potential topological points were identified (Figure 5(c)). 

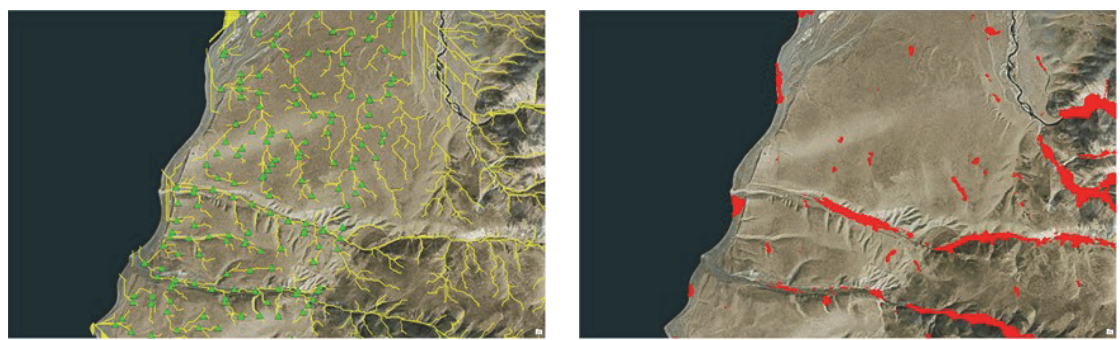

(a) The map before eliminating redun- (b) Difference of elevation image: red areas dancy: yellow lines are extracted valley lines are qualified to be deep valleys. and green triangles are intersections.

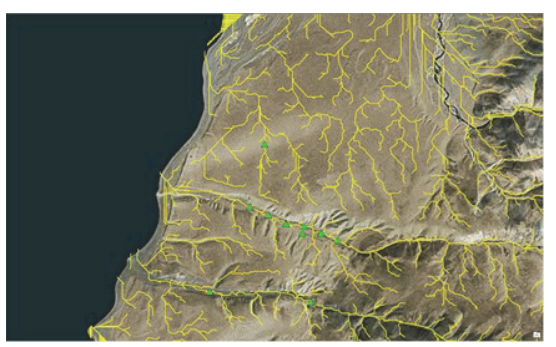

(c) The map after eliminating redundancy: yellow lines are extracted valley lines and green triangles are the redundancy free intersections.

Figure 5: Intermediate map to remove redundant intersections.

Moreover, there are many potential points that are too close to each other and some water bodies have more than one potential point. Thus, only one potential point was selected for each water body among all potential points. For newly built upper reservoir, the point with the smallest slope was selected and the mean slope of all the points around the corresponding lower water body was assigned to the point. For newly built lower reservoir, the point with the minimum distance to the corresponding upper water body was selected.

In addition to topological restriction, the potential points should meet some other criteria which are shown in Table 3 , such as minimum slope and minimum max head between potential points and adjacent lake, to remove unpractical potential points. The remaining potential points can be called potential sites.

\subsection{The estimation of potential PHS storage capacity}

The potential PHS capacity is always dependent upon the potential hydraulic energy available in the upper reservoir, which was estimated as follows [32]:

$$
E=\rho g h V \eta
$$

where: 


$$
\begin{aligned}
E & =\text { energy available (Joules) } \\
\rho & =\text { density }\left(1000 \mathrm{~kg} / \mathrm{m}^{3} \text { for water }\right) \\
g & =\text { acceleration of gravity }\left(9.8 \mathrm{~m} / \mathrm{s}^{2}\right) \\
h & =\text { average head }(\mathrm{m}) \\
V & =\text { volume of upper reservoir } \\
\eta & =\text { generation efficiency (assumed as } 90 \%)
\end{aligned}
$$

Due to the volume of the potential upper reservoir (an existing water body or a new reservoir in the valley), it was hard to calculate accurately and as such only estimations were made in this study. The volume of existing water body under category $\mathrm{T} 1$ was estimated as follows:

$$
V=A d
$$

where:

$\mathrm{A}=$ area of existing lake $(\mathrm{m} 2)$

$\mathrm{d}=$ available depth of existing lake (because the depth of water body was unknown, the depth was assumed to be $20 \mathrm{~m}$, which was equal to the estimated depth of reservoir built on a flat in [33])

The upper reservoir was assumed to be a triangular pyramid under category T2 (Figure 6) and its volume was estimated as follows:

$$
V=\frac{1}{6} l w d
$$

where:

$\mathrm{w}=$ maximum width of upper reservoir (assumed as $300 \mathrm{~m}$ )

$\mathrm{d}=$ maximum depth of upper reservoir (assumed as $70 \mathrm{~m}$, which was equal to the absolute value of maximum difference of elevation in Table 3)

$l=$ maximum length of upper reservoir, which was calculated as follows:

$$
l=\frac{d}{\tan \theta}
$$

$\theta=$ the average slope of valley line (assumed as the slope of potential site point) $=$

\subsection{Explore the distance from potential sites to grid connections}

All potential sites were converted to point features and the distances from them to the nearest grid connections were calculated. A map (Figure 13) that uses graduated colors symbol to display the distances was made to analyze the spatial pattern of distances of potential sites.

\section{Results}

\subsection{Potential under category T1}

The overall theoretical potential storage capacity under category $\mathrm{T} 1$ is about 997.2 GWh and the number of potential sites is 69. The upper or lower lake is usually a barrier lake within a valley. The average elevation of existing lower lakes is $3622 \mathrm{~m}$ and the average elevation of upper lakes is $4455 \mathrm{~m}$. A typical site selection is shown in Figure 7. 


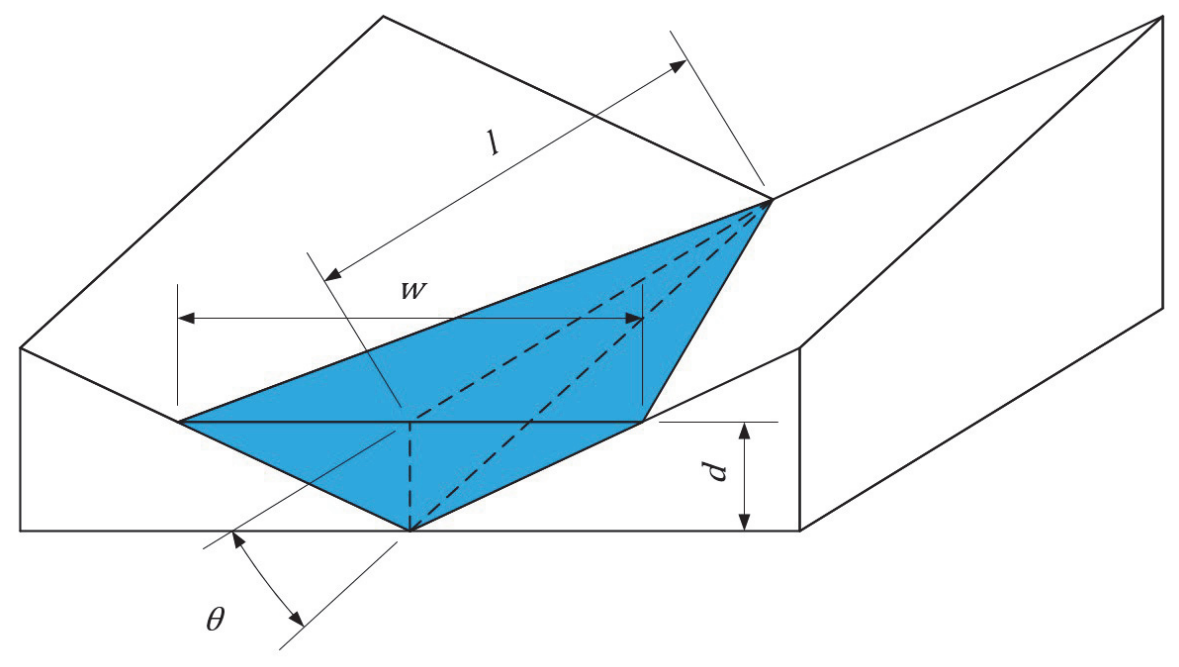

Figure 6: The upper reservoir model. The blue areas are water body and the white areas are hillsides.

The distribution of potential sites (Figure 8) is not dispersed and possible sites with most impressive storage capacity are mainly distributed in Nyingchi, where the topographical changes and natural mountain lake resources are the most abundant in Tibet. Table 4 shows the number and total storage capacity of the theoretical potential for Tibet's seven subareas. Nyingchi and Shigatse are the two subareas, which possess the greatest theoretical potential. However, the other subareas are far less substantial when compared to these two regions.

Table 4: The number and total storage capacity of the theoretical potential for Tibet's seven subareas under $\mathrm{T} 1$

\begin{tabular}{lll}
\hline Name & Count & Storage Capacity $(\mathrm{GWh})$ \\
\hline Ngari & 0 & 0 \\
Nagqu & 1 & 9.7 \\
Chamdo & 5 & 30.5 \\
Nyingchi & 55 & 682.5 \\
Lhasa & 0 & 0 \\
Shigatse & 7 & 268.8 \\
Shannan & 1 & 5.7 \\
Sum & 69 & 997.2 \\
\hline
\end{tabular}




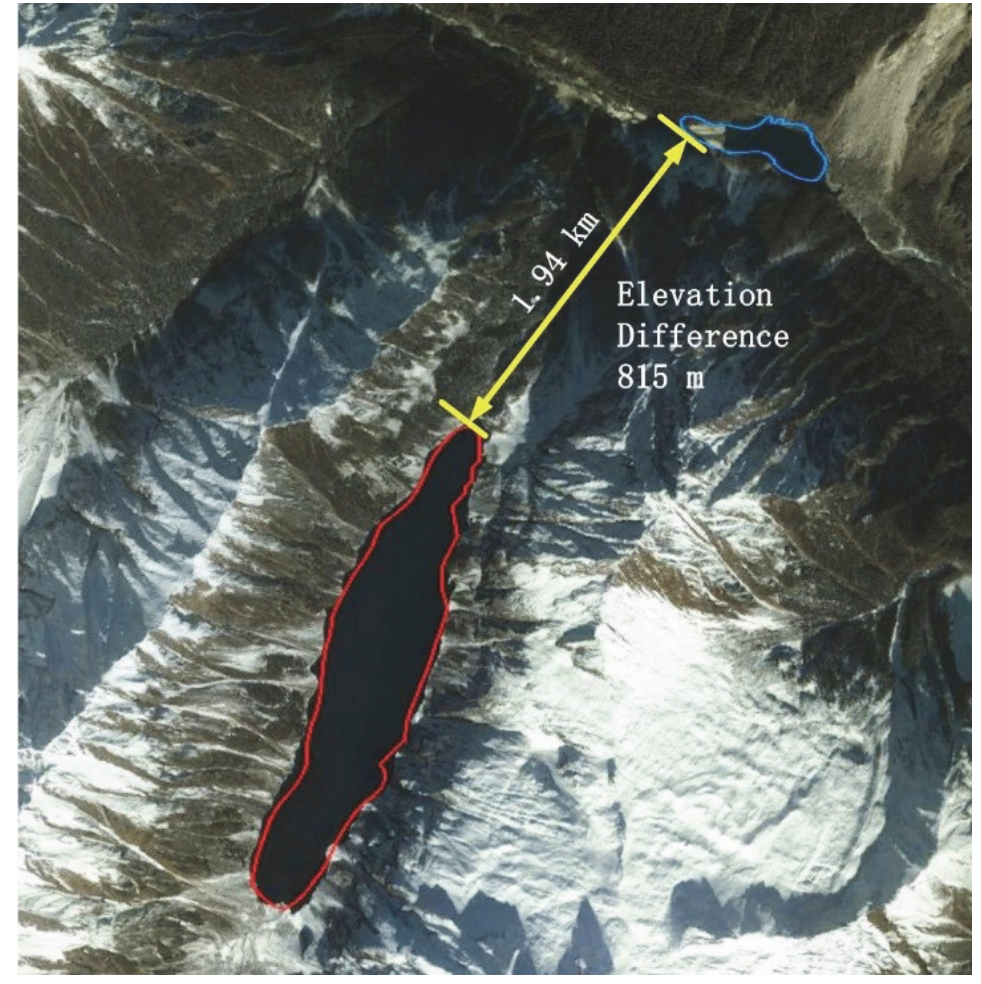

Figure 7: A typical site selection of linking two lakes. The latitude and longitude coordinates of the center point of red lake is $30^{\circ} 04^{\prime} 23^{\prime \prime} \mathrm{N} 94^{\circ} 27^{\prime} 52^{\prime \prime} \mathrm{E}$. The elevation of the red lake is 4115 $\mathrm{m}$. The elevation of the blue lake is $3300 \mathrm{~m}$. The figure was created from Google Earth.

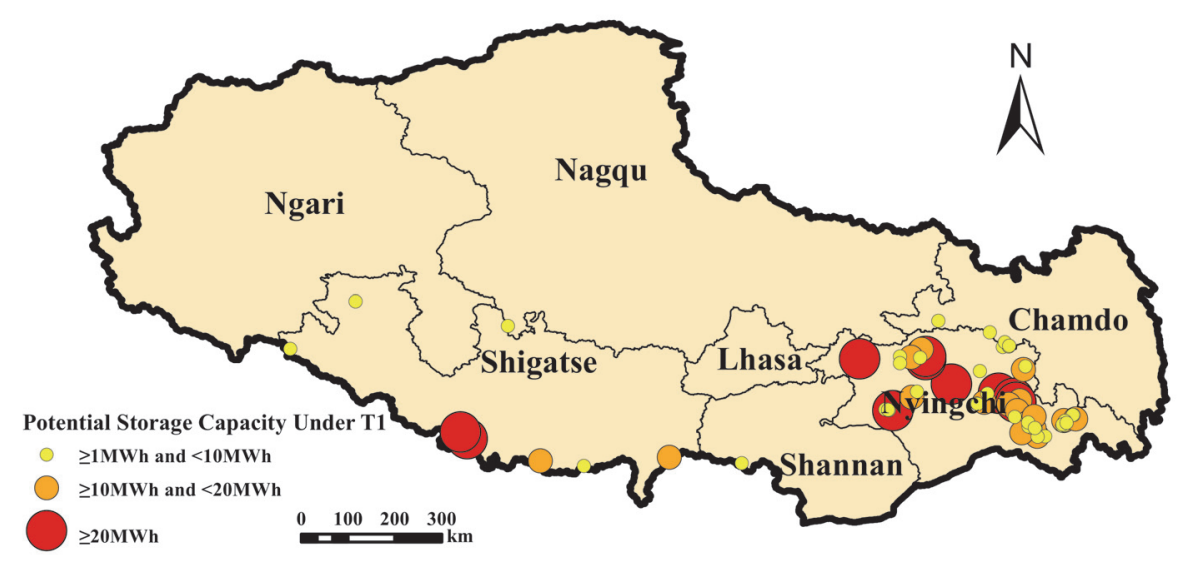

Figure 8: Distribution of potential sites in Tibet under T1 


\subsection{Potential under category T2}

4.2.1. Building new upper reservoirs (hereinafter referred to as category S1)

The overall theoretical potential storage capacity under S1 is about 946.2 GWh and the number of potential sites is 274 . The upper reservoir is always located in a valley without a perennial stream. The average elevation of potential sites is $5043 \mathrm{~m}$ and the average elevation of existing water bodies is 4368 m. A typical site selection is shown in Figure 9.

As shown in Figure 10, potential sites in category S1 is more dispersed than the results under category T1. However, north and northeastern Tibet as well as the area around Lhasa do not have an abundance of potential sites compared to other areas. Table 5 shows the number and storage capacity of the theoretical potential for Tibet's seven subareas. Although Nyingchi still has a lot of potential sites and the largest storage capacity, the potential sites of the other areas also have considerable storage capacity with the exception of Lhasa.

Table 5: The number and total storage capacity of the theoretical potential for Tibet's seven subareas under S1

\begin{tabular}{lll}
\hline Name & Count & Storage Capacity $(\mathrm{GWh})$ \\
\hline Ngari & 42 & 122.5 \\
Nagqu & 34 & 113.0 \\
Chamdo & 32 & 119.2 \\
Nyingchi & 90 & 323.7 \\
Lhasa & 3 & 10.7 \\
Shigatse & 59 & 209.2 \\
Shannan & 14 & 47.9 \\
Sum & 274 & 946.2 \\
\hline
\end{tabular}




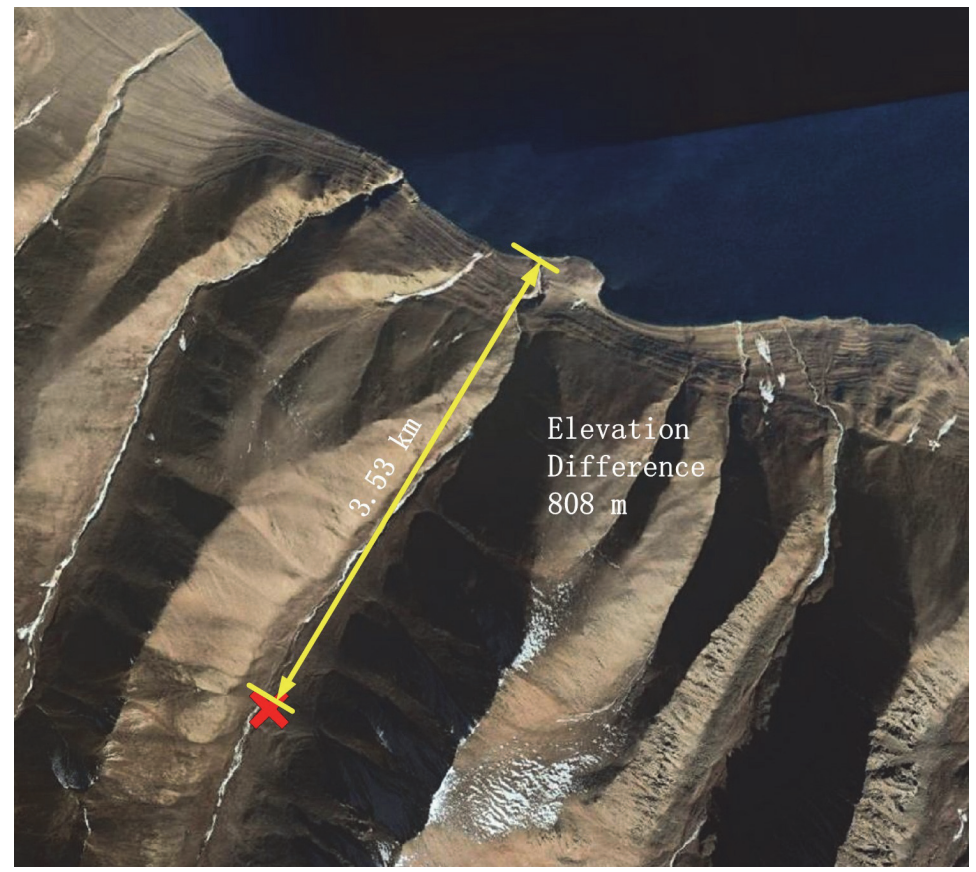

Figure 9: A typical selection result of linking an existing lake and a newly built upper reservoir. The red sign is the specific site selection for a scheme of lower reservoir. The red sign's latitude and longitude coordinates are $30^{\circ} 44^{\prime} 11^{\prime \prime} \mathrm{N} 86^{\circ} 25^{\prime} 20^{\prime \prime} \mathrm{E}$, respectively. The elevation of it is 5348 $\mathrm{m}$. The elevation of the lake is $4540 \mathrm{~m}$. The figure was created from Google Earth.

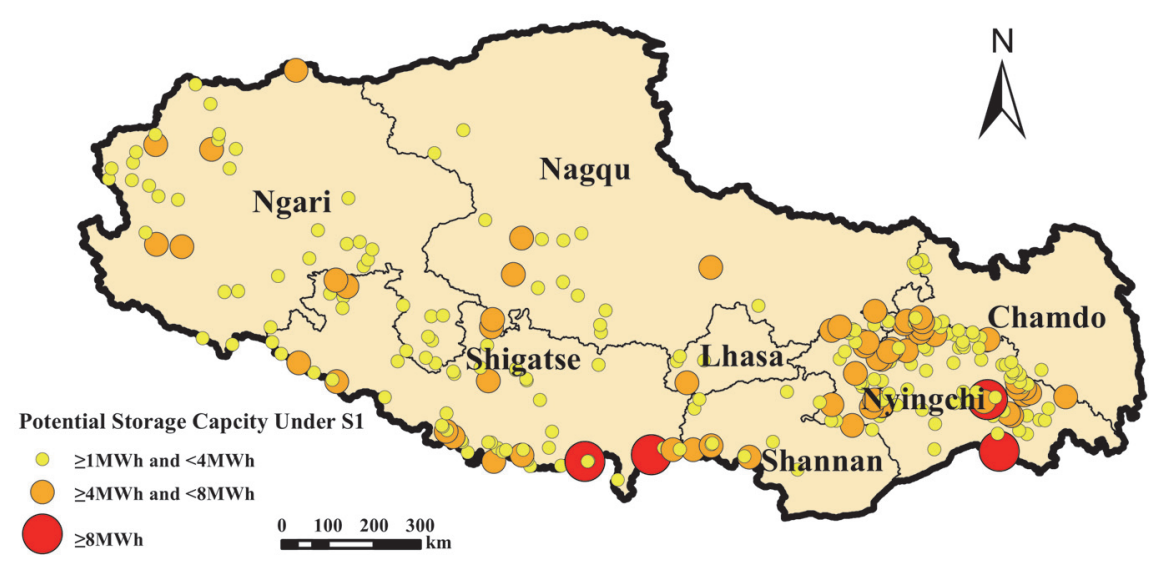

Figure 10: Distribution of potential sites in Tibet under S1 
4.2.2. Building new lower reservoirs (hereinafter referred to as category S2)

The overall theoretical potential storage capacity under category S2 is about $2390.4 \mathrm{GWh}$ and the number of potential sites is 215 . The lower reservoir is usually located in a valley with perennial stream. The average elevation of the potential sites is $3765 \mathrm{~m}$ and average elevation of existing water bodies is 4512 m. A typical site selection is shown in Figure 11.

The distribution of potential sites (Figure 12) is also clustered and most of the potential sites are concentrated in southeastern Tibet. Nyingchi has the most potential sites, followed by Shigatse and Shannan. As shown in Table 3, Nyingchi, Chamdo, Shigatse and Shannan have a considerably high storage capacity.

Table 6: The number and total storage capacity of the theoretical potential for Tibet's seven subareas under $\mathrm{S} 2$

\begin{tabular}{lll}
\hline Name & Count & Storage Capacity (GWh) \\
\hline Ngari & 2 & 23.2 \\
Nagqu & 5 & 24.7 \\
Chamdo & 11 & 514.8 \\
Nyingchi & 131 & 1278.5 \\
Lhasa & 2 & 14.9 \\
Shigatse & 30 & 230.7 \\
Shannan & 34 & 303.6 \\
Sum & 215 & 2390.4 \\
\hline
\end{tabular}




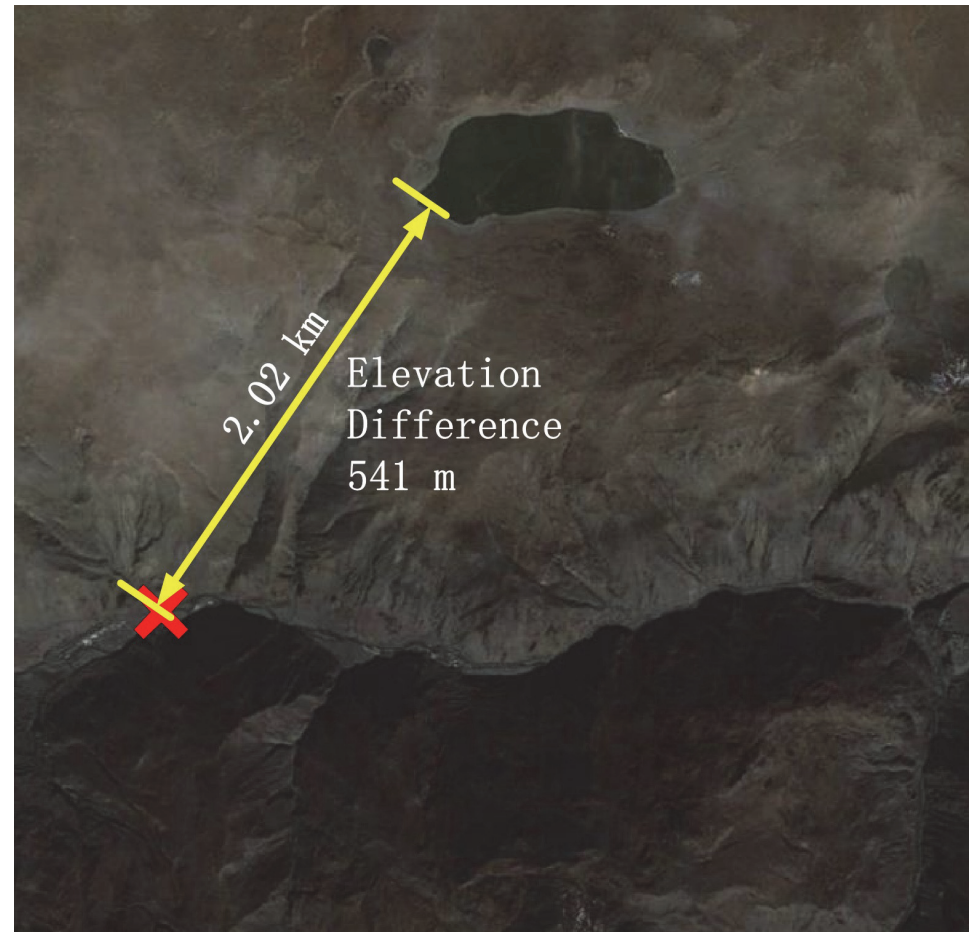

Figure 11: A typical selection result of linking an existing lake and a newly built lower reservoir. The red sign is the specific site selection for a scheme of lower reservoir. The red sign's latitude and longitude coordinates is $29^{\circ} 08^{\prime} 00^{\prime \prime} \mathrm{N} 90^{\circ} 08^{\prime} 24^{\prime \prime} \mathrm{E}$. The elevation of it is 4229 m. The elevation of the upper lake is $4770 \mathrm{~m}$. The figure was created from Google Earth.

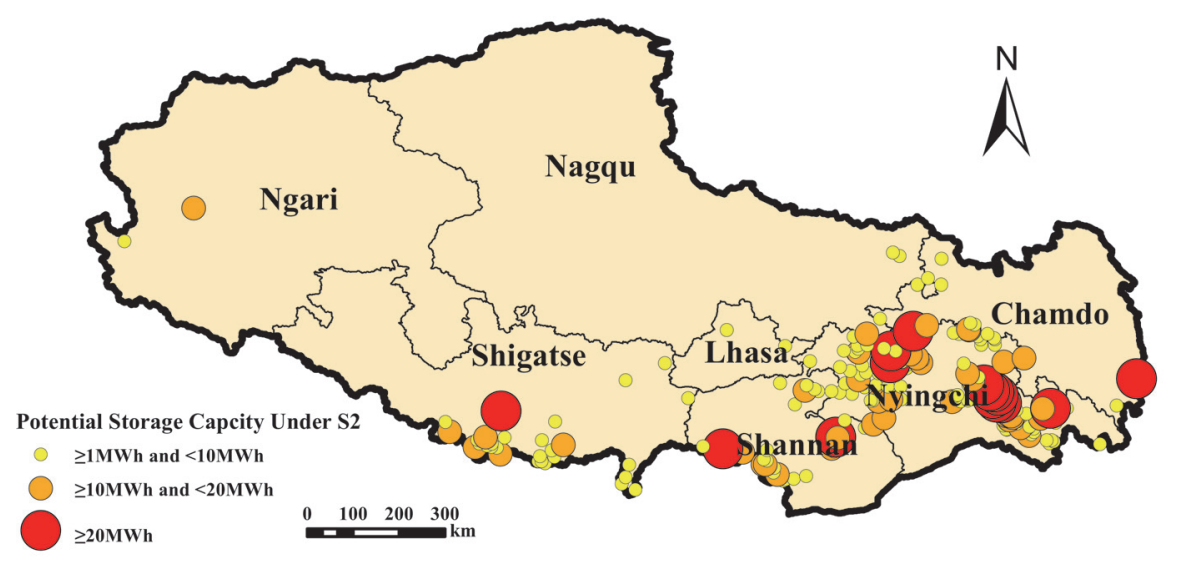

Figure 12: Distribution of potential sites in Tibet under S2 


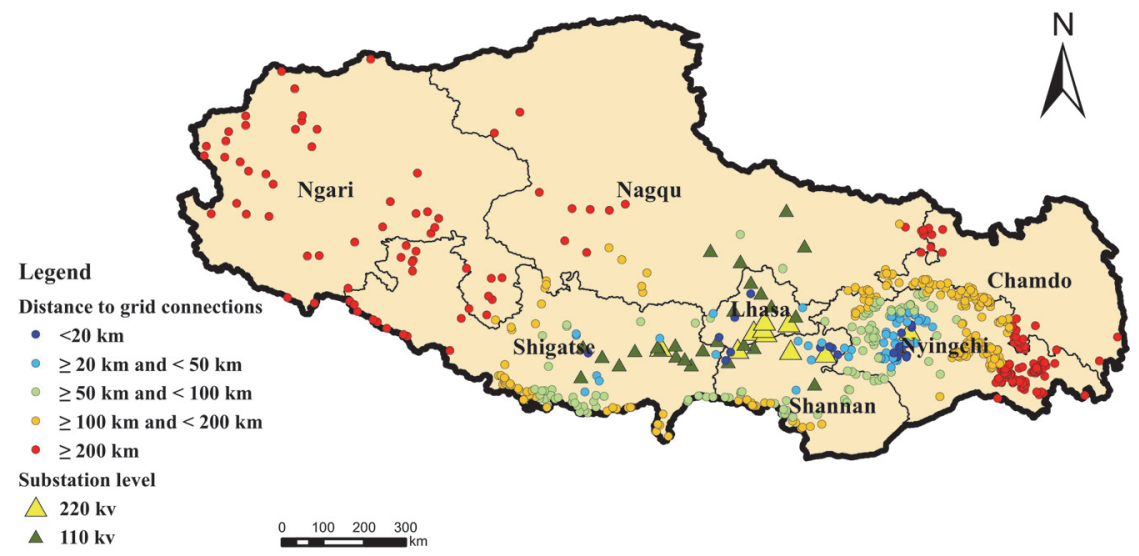

Figure 13: The distances from all potential site points to the nearest substation

Table 7: The frequency and percentage table of the distances from all potential site points to the nearest substation.

\begin{tabular}{lll}
\hline Distance & Frequency & Percentage \\
\hline$<20 \mathrm{~km}$ & 22 & $3.9 \%$ \\
$20-50 \mathrm{~km}$ & 57 & $10.2 \%$ \\
$50-100 \mathrm{~km}$ & 113 & $20.3 \%$ \\
$100-200 \mathrm{~km}$ & 192 & $34.4 \%$ \\
$>200 \mathrm{~km}$ & 174 & $31.2 \%$ \\
\hline
\end{tabular}

As presented in Figure 13, Tibet's substations are mainly located in the central areas, where only a few potential sites exist. The present data in Table 7 indicates that more than $96 \%$ of potential sites have more than $20 \mathrm{~km}$ to their nearest substation and more than $85 \%$ of potential sites have more than $50 \mathrm{~km}$ to their nearest substation. Since $20 \mathrm{~km}$ is the current maximum distance to substations and $50 \mathrm{~km}$ is the original maximum distance to substations in [33], most potential sites are located far away from existing substations. This phenomenon reflects that the potential sites, which have access to a grid connection to build PHS station, are still limited in Tibet currently. Only a few of potential sites located in Shigatse, Lhasa, Shannan, and Nyingchi have an adequate grid infrastructure to build PHS stations. 


\section{Discussions}

\subsection{Results Analysis}

According to Tables 4, 5, 6, the order of theoretical storage capacity of the three situations from largest to smallest is: category S2 (2390.4 GWh), T1 (997.2 GWh), and S1 (946.2 GWh). The total storage capacity under T1 is greater than $\mathrm{S} 1$ and it is much less than S2; the number of sites under T1 (69) is much less than S1 (274) or S2 (215). When the average storage capacity per site is examined, T1 (14.45 GWh) is close to $\mathrm{S} 1(11.12 \mathrm{GWh})$ and both situations are greater than S2 $(3.45 \mathrm{GWh})$. The storage capacity of the Tianhuangping PHS station, a well-known PHS station in China, is 10.46 GWh [42]. Thus, the storage capacity per potential site under T1 and S2 is remarkably good.

Figures 8,10 , and 12 show the spatial distributions of potential sites in the three situations. For T1, the potential sites are mainly located in Nyingchi, which is one of areas with the most abundant rainfall in Tibet [43] and the main terrain of Nyingchi are mountains and valleys. The humid climate, which provides enough rainfall water or glacier melt water, and mountainous terrain might form a lot of block lakes in valleys, so that there are many pairs of lakes in Nyingchi. For S1, the distribution of the potential sites is more dispersed in Tibet than the other two situations. The evenly distributed lakes and the mountainous terrain of Qinghai-Tibet Plateau, which can form an upper reservoir, might be the reason. For S2, the potential sites mainly locate in the east, southeastern, and south of Tibet. Similar to T1, there are lots of block lakes between mountains, which can be upper reservoirs, in these areas. And plenty of rivers and streams form numerous valleys, where people can build a lower reservoir.

If other criteria such as economic and environmental factors were considered, T1 might be more economic and environmentally friendly than T2. For T1, the main constructions are building a new penstock and a new power house (Figure 1) to transform two existing lakes to PHS. For T2, besides building a new penstock and a new power house, it needs to build an additional new reservoir. Thus, $\mathrm{T} 1$ costs less and has smaller environmental impacts than $\mathrm{T} 2$ due to less construction. Only considering the two situations in T2, S1 can be more costly because of its upper reservoirs' high average elevation, which is $1278 \mathrm{~m}$ higher than the average elevation of S2, and lower storage capacity per sites. Although S2's potential sites always locates in a stream valley, where a newly built dam can influence the ecosystem of the stream where a new dam would be built, S2 is comparable with the average elevation and storage capacity per site of $\mathrm{T} 1$ and it is more widely distributed than $\mathrm{T} 1$.

In addition, the substations mainly locate in the central areas of Tibet, so that only a few sites (see Figure 13) in Shigatse, Lhasa, Shannan, and Nyingchi have the enough grid infrastructure to build PHS station. Thus, T1 and S2 might be the practical topologies to build PHS station. 


\subsection{Limitations}

The approaches of assessing the potential have some limitations. The data, such as GDEM and water body data, have flaws in this study. Some areas of GDEM have large elevation errors. The wrong elevation value will result in the wrong valley line extraction and wrong slope. Thus, some potential sites are selected by mistake. The main limitation of the water body data is out of date. Because SRTM depicted the water body in February 2000 at the time of the shuttle flight, the water body data could only show the lakes' extents in February 2000 (winter) rather than nowadays data and also overlooked some relatively small lakes. Thus, the areas of these lakes are underestimated.

GIS models applied in this study only examined two topologies, T1 and T2, which are suitable for potential site selection in Tibet. In fact, there are some other topologies which could be also feasible to build PHS stations in other areas. Even if without considering other topologies and data limits, the new proposed GIS model, which is built by closing a valley with a dam, could not select all the theoretical sites of PHS. Actually, the high place that is ringed on three sides by high mountains could be potential sites. The model only considers the feasibility to build a new reservoir in a narrow valley, but the place in a relatively wide valley is overlooked. Next, the valley lines derived from DEM are not desirable enough. If an algorithm that could extract valley line at high elevation location without introducing a lot of redundant drainage lines, the results of site selection will be more desirable than using hydrology analysis. In addition, most constraints and thresholds are subjective and can be modified to improve the site selection results. For example, reducing the buffer interval, decreasing the maximum head could generate more potential sites.

The storage capacity was roughly estimated. First, the storage capacity only depends on the upper reservoirs' volume. In fact, some lower reservoirs' volume are less than upper reservoirs', so the storage capacity should depend on the volume of lower reservoirs rather than upper one. For example, the total storage capacity of Chamdo is not consistent with the number of potential sites in Table 6, because Chamdo has a very large barrier lake, which can be the upper reservoir. However, the lower reservoir might not be able to have comparable volume to the upper reservoir. Second, the estimation models are defined by subjective assumption, so that the estimated volume of upper reservoir may be inaccurate on a large-scale. However, further work can improve the accuracy of the estimation. For example, using volume calculation tools, such as Surface Volume tool in ArcGIS [44], could get more accurate estimations for potential sites under S1 in the actual individual project.

Other criteria should be considered in the further work. This study only selects the theoretical potential sites and analyzes the distances from potential sites to grid connections. If a series of social, infrastructure, environmental, and economic constraints are considered, a more practically potential for PHS would be selected [33]. In addition, the capacities of solar, and wind power stations, are also important for a hybrid renewable energy system coupled with PHS station, so that these stations should be considered in further site selection studies. 
Apart from the limitation of approaches, the scarcity of available sites for two large reservoirs is not the only technical limitation of PHS. In addition, a long construction period (typically around 10 years), a high capital investment for construction (typically hundreds to thousands of million US dollars) and environmental issues (e.g. clearing trees and others objects from the reservoir area) bring further constraints to the deployment of PHS [15, 45]. In addition, there is only one existing PHS facility in Tibet, it is hard to use existing PHS facilities to validate the result.

Although the results of the research might not be qualified to the accuracy and definition required for actual individual projects due to above-mentioned limitations, this work could be preliminary selection for actual projects in the future.

\section{Acknowledgements}

The authors would like to thank Prof. Yelena Ogneva-Himmelberger, Prof. John Rogan, Mr. Garfield Barclay, and Dongjie Fu who reviewed the draft paper and their comments to improve the study.

The authors would also like to acknowledge the data provided by the United States Geological Survey (USGS), the Ministry of Economy, Trade and Industry of Japan (METI) and the National Aeronautics and Space Administration (NASA).

\section{References}

[1] Q. Wang, H.-N. Qiu, Situation and outlook of solar energy utilization in tibet, china, Renewable and Sustainable Energy Reviews 13 (8) (2009) 2181-2186. doi:10.1016/j.rser.2009.03.011.

[2] Z. Liu, X. Zou, H. Liu, Land desertification monitoring on tibetan plateau using remote sensing technology, in: AGU Fall Meeting Abstracts, Vol. 1, 2012, p. 0518.

URL http://adsabs . harvard. edu/abs/2012AGUFM. B33B0518L

[3] G. office of the state council of the people's republic of China, Energy development strategy action plan from 2014 to 2020 (2014). URL http://www.gov.cn/zhengce/content/2014-11/19/content_ 9222.htm

[4] N. E. A. of China, The brief introduction of the status quo of photovoltaic construction in the first half of 2015 (2015-07-28).

URL http://www.nea.gov.cn/2015-07/28/c_134455530.htm

[5] T. E. Administration, Tibet's installed power-generating capacity is close to 1.7 million kilowatts and the growth rate is $74.2 \%$ in the last four years (2015-07-16).

URL http://www.xizang.gov.cn/xzshfz/90284.jhtml 
[6] P. Zhang, W. Li, S. Li, Y. Wang, W. Xiao, Reliability assessment of photovoltaic power systems: Review of current status and future perspectives, Applied Energy 104 (2013) 822-833. doi:10.1016/j . apenergy .2012.12. 010.

[7] T. Kousksou, P. Bruel, A. Jamil, T. El Rhafiki, Y. Zeraouli, Energy storage: Applications and challenges, Solar Energy Materials and Solar Cells 120 (2014) 59-80. doi:10.1016/j.solmat.2013.08.015.

[8] S. Kucukali, Finding the most suitable existing hydropower reservoirs for the development of pumped-storage schemes: An integrated approach, Renewable and Sustainable Energy Reviews 37 (2014) 502-508. doi:10.1016/j.rser.2014.05.052.

URL http://www.sciencedirect.com/science/article/pii/ S1364032114003773

[9] B. Zakeri, S. Syri, Electrical energy storage systems: A comparative life cycle cost analysis, Renewable and Sustainable Energy Reviews 42 (2015) 569-596. doi:10.1016/j.rser.2014.10.011.

[10] X. Luo, J. Wang, M. Dooner, J. Clarke, Overview of current development in electrical energy storage technologies and the application potential in power system operation, Applied Energy 137 (2015) 511-536. doi:10. $1016 / j$. apenergy . 2014.09.081.

[11] S. Rehman, L. M. Al-Hadhrami, M. M. Alam, Pumped hydro energy storage system: A technological review, Renewable and Sustainable Energy Reviews 44 (2015) 586-598.

[12] A. Evans, V. Strezov, T. J. Evans, Assessment of utility energy storage options for increased renewable energy penetration, Renewable and Sustainable Energy Reviews 16 (6) (2012) 4141-4147. doi:10.1016/j.rser. 2012.03 .048$.

[13] W. A. Omran, M. Kazerani, M. Salama, Investigation of methods for reduction of power fluctuations generated from large grid-connected photovoltaic systems, Energy Conversion, IEEE Transactions on 26 (1) (2011) 318-327.

[14] M. Black, G. Strbac, Value of bulk energy storage for managing wind power fluctuations, Energy conversion, ieee transactions on 22 (1) (2007) 197-205.

[15] P. Denholm, G. L. Kulcinski, Life cycle energy requirements and greenhouse gas emissions from large scale energy storage systems, Energy Conversion and Management 45 (13) (2004) 2153-2172.

[16] T. Ma, H. Yang, L. Lin, J. Peng, Pumped storage-based standalone photovoltaic power generation system: Modeling and techno-economic optimization, Applied Energy 137 (2015) 649-659. 
[17] M. Tao, H. Yang, L. Lin, Feasibility study and economic analysis of pumped hydro storage and battery storage for a renewable energy powered island, Energy Conversion and Management 79 (3) (2014) 387-397.

[18] J. Margeta, Z. Glasnovic, Theoretical settings of photovoltaic-hydro energy system for sustainable energy production, Solar Energy 86 (3) (2012) 972982.

[19] J. Margeta, Z. Glasnovic, Introduction of pv energy into an existing hep, IEEE Transactions on Energy Conversion 26 (3) (2011) 717-727.

[20] Margeta, J. Glasnović, Zvonimir, The role of water-energy storage in the pv-psh power plant development, Journal of Energy Engineering.

[21] J. Margeta, Z. Glasnovic, Feasibility of the green energy production by hybrid solar + hydro power system in europe and similar climate areas, Renewable and Sustainable Energy Reviews 14 (6) (2010) 1580-1590.

[22] N. Development, R. Commission, Notice on the related issues of improving price formation mechanism of pumped storage power station (2014).

URL http://jgs.ndrc.gov.cn/zcfg/201408/t20140812_622012.html

[23] N. Development, R. Commission, The national development and reform commission's views on issues relating to promote pumped storage hydropower station development orderly and healthily (2014).

URL http://www.sdpc.gov.cn/gzdt/201411/t20141117_648312.html

[24] T. central committee of the communist party of China, S. Council, Several opinions on further deepening the reform of electric power system (2015).

[25] Z. Ming, Z. Kun, L. Daoxin, Overall review of pumped-hydro energy storage in china: Status quo, operation mechanism and policy barriers, Renewable and Sustainable Energy Reviews 17 (2013) 35-43.

[26] Z. Ming, F. Junjie, X. Song, W. Zhijie, Z. Xiaoli, W. Yuejin, Development of china's pumped storage plant and related policy analysis, Energy Policy 61 (2013) 104-113. doi:10.1016/j .enpol.2013.06.061.

[27] T. P. G. of Qinghai Province, The brief introduction of qinghai-tibet grid interconnection project (2011-11-30).

URL http://news.xinhuanet.com/energy/2011-11/30/c_122356686. htm

[28] T. P. G. of Qinghai Province, Qinghai-tibet grid interconnection project makes tibet's electric power delivery become the norm (2015-10-31).

URL http://www.qh.gov.cn/zwgk/system/2015/10/31/010185905. shtml

[29] T. C. G. of China, Sichuan-tibet grid interconnection will be completed to connect tibet's isolate grid (2014-08-04).

URL http://www.gov.cn/xinwen/2014-08/04/content_2729497.htm 
[30] H. Chen, T. N. Cong, W. Yang, C. Tan, Y. Li, Y. Ding, Progress in electrical energy storage system: A critical review, Progress in Natural Science 19 (3) (2009) 291-312. doi:10.1016/j.pnsc.2008.07.014.

[31] D. Connolly, S. MacLaughlin, M. Leahy, Development of a computer program to locate potential sites for pumped hydroelectric energy storage, Energy 35 (1) (2010) 375-381. doi:10.1016/j.energy.2009.10.004.

[32] N. Fitzgerald, R. Lacal Arántegui, E. McKeogh, P. Leahy, A gis-based model to calculate the potential for transforming conventional hydropower schemes and non-hydro reservoirs to pumped hydropower schemes, Energy 41 (1) (2012) 483-490. doi:10.1016/j.energy.2012.02.044.

[33] M. Gimeno-Gutiérrez, R. Lacal-Arántegui, Assessment of the european potential for pumped hydropower energy storage (2013).

[34] D. Hall, R. Lee, Assessment of opportunities for new united states pumped storage hydroelectric plants using existing water features as auxiliary reservoirs, Report, Idaho National Laboratory (INL) (2014).

[35] M. Gimeno-Gutiérrez, R. Lacal-Arántegui, Assessment of the european potential for pumped hydropower energy storage based on two existing reservoirs, Renewable Energy 75 (2015) 856-868.

[36] NASA, Aster global digital elevation map announcement. URL http://asterweb.jpl.nasa.gov/gdem.asp

[37] T. Tachikawa, M. Hato, M. Kaku, A. Iwasaki, Characteristics of aster gdem version 2, in: Geoscience and Remote Sensing Symposium (IGARSS), 2011 IEEE International, IEEE, 2011, pp. 3657-3660.

[38] A. G. V. Team, Aster global digital elevation model version 2 - summary of validation results.

URL http://www.jspacesystems.or.jp/ersdac/GDEM/ ver2Validation/Summary_GDEM2_validation_report_final.pdf

[39] USGS, Srtm water body dataset. URL https://1ta.cr.usgs.gov/srtm_water_body_dataset

[40] ESRI, Hydrologic analysis sample applications (11/8/2012 2012). URL http://resources.arcgis.com/en/help/main/10.1/index.html\# //009z0000005z000000

[41] A. M. López, F. Lumbreras, J. Serrat, J. J. Villanueva, Evaluation of methods for ridge and valley detection, IEEE Transactions on Pattern Analysis and Machine Intelligence 21 (4) (1999) 327-335.

[42] D. Hua, Brief introduction of tianhuangping pumped storage power station, Energy Research and Information 1 (1994) 007. 
[43] X. Liu, D. Qin, X. Shao, L. Zhao, T. Chen, J. Ren, Variation and abrupt change of precipitation in nyingchi prefecture of tibet autonomous region in past 350 years, Journal of Glaciology and Geocryology 25 (4) (2003) $375-379$.

[44] ESRI, Surface volume (3d analyst) (11/8/2012).

URL http://resources.arcgis.com/en/help/main/10.1/index.html\# //00q900000027000000

[45] P. Denholm, T. Holloway, Improved accounting of emissions from utility energy storage system operation, Environmental science \& technology 39 (23) (2005) 9016-9022. 